\title{
Research Article \\ On the Stability of Some Discrete Fractional Nonautonomous Systems
}

\author{
Fahd Jarad, 1 Thabet Abdeljawad, ${ }^{1}$ \\ Dumitru Baleanu, ${ }^{1,2}$ and Kübra Biçen ${ }^{1}$ \\ ${ }^{1}$ Department of Mathematics and Computer Sciences, Faculty of Arts and Sciences, Çankaya University, \\ 06530 Ankara, Turkey \\ ${ }^{2}$ Institute for Space Sciences, P.O. Box MG-23, 76900 Magurele-Bucharest, Romania
}

Correspondence should be addressed to Dumitru Baleanu, dumitru@cankaya.edu.tr

Received 7 June 2011; Revised 28 November 2011; Accepted 29 November 2011

Academic Editor: Ibrahim Sadek

Copyright (C) 2012 Fahd Jarad et al. This is an open access article distributed under the Creative Commons Attribution License, which permits unrestricted use, distribution, and reproduction in any medium, provided the original work is properly cited.

Using the Lyapunov direct method, the stability of discrete nonautonomous systems within the frame of the Caputo fractional difference is studied. The conditions for uniform stability, uniform asymptotic stability, and uniform global stability are discussed.

\section{Introduction}

The fractional calculus which is as old as the usual calculus deals with the generalization of integration and differentiation of integer order to any order. Recently, there has been great interest in this calculus as it turned out that it has many applications in many fields of science and engineering [1-6].

The analogous theory for discrete fractional calculus was initiated by Miller and Ross in [7]. Building this theory was continued in [8-12].

The stability of fractional-order linear and nonlinear dynamic systems was a subject of many reports [13-19], while the stability of discrete dynamic systems was discussed in many books and articles (see, e.g., [20] and the references therein). But the stability of discrete fractional dynamic systems, to our knowledge, has not been reported.

The analysis of the dynamics of complex or hypercomplex systems requires new methods and techniques to be developed. Particularly, the systems which have both continuous evolution and discrete evolution represents a new direction in many fields of science and engineering. For these type of systems the classical or the improved techniques and methods (e.g., with or without nonlocal terms described usually by the fractional 
derivatives and integrals) are not enough to describe accurately their dynamics. In this line of thought the combination of the fractional and discrete operators leads to a better description of the systems mentioned above. However, these new hybrid methods are at the beginning of their evolutions and new efforts are required to be developed and implemented in practical applications.

In [19], to demonstrate the advantage of using fractional-order derivatives instead of integer-order derivatives, the authors considered 2 systems. The system with integer-order derivative turned to be unstable. But the second system, where the integer-order derivative was replaced by fractional-order derivative, turned out to be stable. The same argument still holds for integer difference and fractional difference systems. To illustrate this, we consider the following two systems:

$$
\begin{gathered}
\Delta x(t)=\mu t^{(\mu-1)} \quad 0<\mu<1 x(0)=x_{0}, \\
{ }^{C} \Delta_{a}^{\alpha} x(t)=\mu t^{(\mu-1)} \quad 0<\alpha<1, a=1-\alpha x(a)=x_{0}, t \in \mathbb{N}_{0},
\end{gathered}
$$

where $t^{(\mu)}=\Gamma(t+1) / \Gamma(t+1-\mu)$ and the operator ${ }^{C} \Delta_{a}^{\alpha}$ is defined by (2.10). The solution of (1.1) is $x(t)=t^{(\mu)}+x_{0}$ which clearly tends to $\infty$ as $t \rightarrow \infty$ for $0<\mu<1$ and thus is unstable. The solution of (1.2) which is defined on $\mathbb{N}_{\alpha}$ has the form

$$
x(t)=x(a)+\mu \Delta_{0}^{-\alpha} t^{(\mu-1)},
$$

where the operator $\Delta_{0}^{-\alpha}$ is defined by (2.6). The value of $\Delta_{0}^{-\alpha} t^{(\mu-1)}$ at $t=\alpha+k \in \mathbb{N}_{\alpha}$ is computed as follows:

$$
\begin{aligned}
\left(\Delta_{0}^{-\alpha} t^{(\mu-1)}\right)(\alpha+k)= & \frac{1}{\Gamma(\alpha)} \sum_{s=0}^{k}(\alpha+k-s-1)^{(\alpha-1)} s^{(\mu-1)} \\
= & \frac{1}{\Gamma(\alpha)} \sum_{s=1}^{k} \frac{\Gamma(\alpha+k-s)}{\Gamma(k-s+1)} \frac{\Gamma(s+1)}{\Gamma(s-\mu+2)} \\
= & \frac{1}{\Gamma(\alpha)}\left[\frac{\Gamma(\alpha+k-1)}{\Gamma(k)} \frac{\Gamma(2)}{\Gamma(3-\mu)}+\frac{\Gamma(\alpha+k)}{\Gamma(k+2)} \frac{\Gamma(3)}{\Gamma(3-\mu)}\right. \\
& \left.+\cdots+\frac{\Gamma(\alpha)}{\Gamma(1)} \frac{\Gamma(k+1)}{\Gamma(k+2-\mu)}\right] .
\end{aligned}
$$

The quantity in (1.4) $\rightarrow 0$ as $k \rightarrow \infty$, and thus the solution (1.3) of (1.2) $\rightarrow x_{0}$ for $0<\mu<1$ as $t \rightarrow \infty$ and therefore is stable.

The purpose of this paper is to state the stability theorem for discrete nonlinear dynamic systems in the sense of the Caputo fractional differences. 
The paper is organized as follows. In Section 2 basic definitions of fractional calculus and discrete fractional calculus are mentioned. Section 3 presents our main results on the stability of discrete fractional nonautonomous systems.

\section{Preliminaries}

For a function $f$ defined on the interval $[a, b]$, the left and right Riemann-Liouville integrals of order $\alpha \in \mathbb{C}, \operatorname{Re}(\alpha)>0$, are defined, respectively, by [1-3]

$$
\begin{aligned}
& \left({ }_{a} I^{\alpha} f\right)(t)=\frac{1}{\Gamma(\alpha)} \int_{a}^{t} \frac{f(s)}{(t-s)^{1-\alpha}} d s, \\
& \left(I_{b}^{\alpha} f\right)(t)=\frac{1}{\Gamma(\alpha)} \int_{t}^{b} \frac{f(s)}{(s-t)^{1-\alpha}} d s,
\end{aligned}
$$

while the left and right Riemann-Liouville derivatives of order $\alpha$ are defined, respectively, by $[1-3]$

$$
\begin{aligned}
& \left({ }_{a} D^{\alpha} f\right)(t)=\left(\frac{d}{d t}\right)^{n}\left({ }_{a} I^{n-\alpha} f\right)(t)=\left(\frac{d}{d t}\right)^{n} \frac{1}{\Gamma(n-\alpha)} \int_{a}^{t} \frac{f(s)}{(t-s)^{1-n+\alpha}} d s \\
& \left(D_{b}^{\alpha} f\right)(t)=\left(-\frac{d}{d t}\right)^{n}\left(I_{b}^{n-\alpha} f\right)(t)=\left(-\frac{d}{d t}\right)^{n} \frac{1}{\Gamma(n-\alpha)} \int_{t}^{b} \frac{f(s)}{(s-t)^{1-n+\alpha}} d s
\end{aligned}
$$

where $n=[\operatorname{Re}(\alpha)]+1$ and $\Gamma(\cdot)$ is the Gamma function.

The left and right Caputo fractional derivatives of order $\alpha$ on the interval $[a, b]$ are defined, respectively, by

$$
\begin{aligned}
& \left({ }_{a}^{C} D^{\alpha} f\right)(t)=\left({ }_{a} I^{n-\alpha} f^{(n)}\right)(t)=\frac{1}{\Gamma(n-\alpha)} \int_{a}^{t} \frac{f^{(n)}(s)}{(t-s)^{1-\alpha}} d s, \\
& \left({ }^{C} D_{b}^{\alpha} f\right)(t)=\left(I_{b}^{n-\alpha} f^{(n)}\right)(t)=\frac{1}{\Gamma(n-\alpha)} \int_{t}^{b} \frac{f^{(n)}(s)}{(s-t)^{1-\alpha}} d s .
\end{aligned}
$$

Some properties of the Riemann-Liouville and Caputo fractional derivatives are stated below [1-3].

Property 1. We have

$$
\begin{aligned}
& \left({ }_{a}^{C} D^{\alpha} f\right)(t)=\left({ }_{a} D^{\alpha} f\right)(t)-\sum_{k=0}^{n-1} \frac{f^{(k)}(a)}{\Gamma(k-\alpha+1)}(t-a)^{k-\alpha}, \\
& \left({ }^{C} D_{b}^{\alpha} f\right)(t)=\left(D_{b}^{\alpha} f\right)(t)-\sum_{k=0}^{n-1} \frac{(-1)^{k} f^{(k)}(b)}{\Gamma(k-\alpha+1)}(b-t)^{k-\alpha} .
\end{aligned}
$$


Property 2. If $f \in A C^{n}[a, b]$ or $C^{n}[a, b]$, then

$$
\begin{aligned}
& \left({ }_{a} I_{a}^{\alpha}{ }_{a}^{C} D^{\alpha} f\right)(t)=f(t)-\sum_{k=0}^{n-1} \frac{f^{(k)}(a)}{\Gamma(k-\alpha+1)}(t-a)^{k-\alpha}, \\
& \left(I_{b}^{\alpha}{ }^{C} D_{b}^{\alpha} f\right)(t)=f(t)-\sum_{k=0}^{n-1} \frac{(-1)^{k} f^{(k)}(b)}{\Gamma(k-\alpha+1)}(b-t)^{k-\alpha} .
\end{aligned}
$$

Below we present basic definitions and properties of fractional sums and differences.

First we define the set $\mathbb{N}_{a}=\{a, a+1, a+2, \ldots\}$ and ${ }_{b} \mathbb{N}=b, b-1, b-2, \ldots$. The (left) fractional sum of $f$, defined on $\mathbb{N}_{a}$, of order $\alpha$ is defined by [7-12]

$$
\Delta_{a}^{-\alpha} f(t)=\frac{1}{\Gamma(\alpha)} \sum_{s=a}^{t-\alpha}(t-\sigma(s))^{(\alpha-1)} f(s)
$$

where $\alpha>0, \sigma(s)=s+1$, and $t^{(\alpha)}=\Gamma(t+1) / \Gamma(t+1-\alpha)$. The (right) fractional sum of $f$, defined on ${ }_{b} \mathbb{N}$, of order $\alpha$ is defined by

$$
\nabla_{b}^{-\alpha} f(t)=\frac{1}{\Gamma(\alpha)} \sum_{s=t+\alpha}^{b}(\rho(s)-t)^{(\alpha-1)} f(s)
$$

where $\rho(s)=s-1$. Analogous of the case of fractional derivatives, the Riemann left and right fractional differences of order $\alpha$ are defined, respectively, by

$$
\begin{gathered}
\Delta_{a}^{\alpha} f(t)=\Delta^{n} \Delta_{a}^{-(n-\alpha)} f(t)=\Delta^{n}\left[\frac{1}{\Gamma(n-\alpha)} \sum_{s=a}^{t-n+\alpha}(t-\sigma(s))^{(n-\alpha-1)} f(s)\right] \\
\nabla_{b}^{\alpha} f(t)=(-1)^{n} \nabla^{n} \nabla_{b}^{-(n-\alpha)} f(t)=(-1)^{n} \nabla^{n}\left[\frac{1}{\Gamma(n-\alpha)} \sum_{s=t+n-\alpha}^{b}(\rho(s)-t)^{(n-\alpha-1)} f(s)\right],
\end{gathered}
$$

where $n=[\alpha]+1$. It can be obviously noticed that $\Delta_{a}^{-\alpha}$ maps functions defined on $\mathbb{N}_{a}$ to functions defined on $\mathbb{N}_{a+\alpha}$ and $\Delta_{a}^{\alpha}$ maps functions defined on $\mathbb{N}_{a}$ to functions defined on $\mathbb{N}_{a+n-\alpha}$, while $\nabla_{b}^{-\alpha}$ maps functions defined on ${ }_{b} \mathbb{N}$ to functions defined on ${ }_{b-\alpha} \mathbb{N}_{a+\alpha}$ and $\nabla_{b}^{\alpha}$ maps functions defined on ${ }_{b} \mathbb{N}$ to functions defined on ${ }_{b-n+\alpha} \mathbb{N}$.

Similar to the case of fractional derivatives, the left and right Caputo fractional differences of order $\alpha$ of a function defined on $\mathbb{N}_{a}$ and ${ }_{b} \mathbb{N}$ are defined, respectively, by [12]

$$
\begin{gathered}
{ }^{C} \Delta_{a}^{\alpha} f(t)=\Delta_{a}^{-(n-\alpha)} \Delta^{n} f(t)=\frac{1}{\Gamma(n-\alpha)} \sum_{s=a}^{t-n+\alpha}(t-\sigma(s))^{(n-\alpha-1)} \Delta^{n} f(s), \\
{ }^{C} \nabla_{b}^{\alpha} f(t)=(-1)^{n} \nabla_{b}^{-(n-\alpha)} \nabla^{n} f(t)=\frac{(-1)^{n}}{\Gamma(n-\alpha)} \sum_{s=t+n-\alpha}^{b}(\rho(s)-t)^{(n-\alpha-1)} \nabla^{n} f(s),
\end{gathered}
$$

where $n=[\alpha]+1$. The following property gives the relation between the Riemann and Caputo fractional differences [12]. 
Property 3. For any $\alpha>0$, we have the following:

$$
\begin{gathered}
{ }^{C} \Delta_{a}^{\alpha} f(t)=\Delta_{a}^{\alpha} f(t)-\sum_{k=0}^{n-1} \frac{(t-a)^{(k-\alpha)}}{\Gamma(k-\alpha+1)} \Delta^{k} f(a), \\
{ }^{C} \nabla_{b}^{\alpha} f(t)=\nabla_{b}^{\alpha} f(t)-\sum_{k=0}^{n-1} \frac{(b-t)^{(k-\alpha)}}{\Gamma(k-\alpha+1)}(-1)^{k} \nabla^{k} f(b) .
\end{gathered}
$$

Particularly, when $0<\alpha<1$ we have

$$
\begin{aligned}
& { }^{C} \Delta_{a}^{\alpha} f(t)=\Delta_{a}^{\alpha} f(t)-\frac{(t-a)^{(-\alpha)}}{\Gamma(1-\alpha)} f(a), \\
& { }^{C} \nabla_{b}^{\alpha} f(t)=\nabla_{b}^{\alpha} f(t)-\frac{(b-t)^{(-\alpha)}}{\Gamma(1-\alpha)} f(b) .
\end{aligned}
$$

Property 4. Let $\alpha>0$, and let $f$ be a function defined on suitable subsets of $\mathbb{N}_{a}$ and ${ }_{b} \mathbb{N}$. Then,

$$
\begin{gathered}
\Delta_{a+n-\alpha}^{-\alpha}{ }^{C} \Delta_{a}^{\alpha} f(t)=f(t)-\sum_{k=0}^{n-1} \frac{(t-a)^{(k)}}{k !} \Delta^{k} f(a), \\
\nabla_{b-n+\alpha}^{-\alpha}{ }^{C} \nabla_{b}^{\alpha} f(t)=f(t)-\sum_{k=0}^{n-1} \frac{(b-t)^{(k)}}{k !}(-1)^{k} \nabla^{k} f(b) .
\end{gathered}
$$

Particularly, when $0<\alpha \leq 1$ we have

$$
\Delta_{a+1-\alpha}^{-\alpha}{ }^{C} \Delta_{a}^{\alpha} f(t)=f(t)-f(a), \quad \nabla_{b-1+\alpha}^{-\alpha}{ }^{C} \nabla_{b}^{\alpha} f(t)=f(t)-f(b) .
$$

\section{Lyapunov Stability Theorems for Nonautonomous Systems of Some Fractional Difference Equations}

The stability theory of dynamic systems has a crucial role within the system theory and engineering. The stability of equilibrium points is usually done by utilizing the Lyapunov stability. Hypercomplex systems, namely, systems with both discrete and continuous behavior, need new methods and techniques to define their Lyapunov stability.

In this section we extend the method of the Lyapunov functions to study the stability of solutions of the following system:

$$
{ }^{\mathrm{C}} \Delta_{t_{0}}^{\alpha} x(t)=g(t+\alpha-1, x(t+\alpha-1)) \quad x\left(t_{0}\right)=x_{0}
$$

where $t_{0}=a+n_{0} \in \mathbb{N}_{a}\left(n_{0} \in \mathbb{N}\right), t \in \mathbb{N}_{n_{0}}, a=\alpha-1, g: \mathbb{N}_{a} \times \mathbb{R}^{n} \rightarrow \mathbb{R}^{n}$ is continuous, and $0<\alpha \leq 1$. We note that a Lyapunov function $V$ for the system (3.1) must depend on $t$ and $x$. Now let $g(t, 0)=0$, for all $t \in \mathbb{N}_{a}$ so that the system (3.1) admits the trivial solution. Next we list some definitions that will be used in studying the stability properties of (3.1). 
Definition 3.1. The trivial solution $x(t)=0$ of (3.1) is said to be

(i) stable if, for each $\epsilon>0$ and $t_{0} \in \mathbb{N}_{a}$, there exists a $\delta=\delta\left(\epsilon, t_{0}\right)>0$ such that for any solution $x(t)=x\left(t, t_{0}, x_{0}\right)$ with $\left\|x_{0}\right\|<\delta$ one has $\|x(t)\|<\epsilon$, for all $t \in \mathbb{N}_{t_{0}} \subseteq \mathbb{N}_{a}$,

(ii) uniformly stable if it is stable and $\delta$ depends solely on $\epsilon$,

(iii) asymptotically stable if it is stable and for all $t_{0} \in \mathbb{N}_{a}$ there exists $\delta=\delta\left(t_{0}\right)>0$ if $\left\|x_{0}\right\|<\delta$ implies that $\lim _{t \rightarrow \infty} x\left(t, t_{0}, x_{0}\right)=0$.

(iv) uniformly asymptotically stable if it is uniformly stable and, for each $\epsilon>0$, there exists $T=T(\epsilon) \in \mathbb{N}_{0}$ and $\delta_{0}>0$ such that $\left\|x_{0}\right\|<\delta_{0}$ implies $\|x(t)\|<\epsilon$ for all $t \in \mathbb{N}_{t_{0}+T}$ and for all $t_{0} \in \mathbb{N}_{a}$,

(v) globally asymptotically stable if it is asymptotically stable for all $x_{0} \in \mathbb{R}^{n}$,

(vi) globally uniformly asymptotically stable if it is uniformly asymptotically stable for all $x_{0} \in \mathbb{R}^{n}$.

Definition 3.2. A function $\phi(r)$ is said to belong to the class $\mathcal{K}$ if and only if $\phi \in C\left[[0, \rho), \mathbb{R}_{+}\right]$, $\phi(0)=0$, and $\phi(r)$ is strictly monotonically increasing in $r$. If $\phi: \mathbb{R}_{+} \rightarrow \mathbb{R}_{+}, \phi \in \mathcal{K}$, and $\lim _{r \rightarrow \infty} \phi(r)=\infty$, then $\phi$ is said to belong to class $\mathcal{K} R$.

Definition 3.3. A real valued function $V(t, x)$ defined on $\mathbb{N}_{a} \times S_{\rho}$, where $S_{\rho}=\left\{x \in \mathbb{R}^{n}:\|x\| \leq\right.$ $\rho\}$, is said to be positive definite if and only if $V(t, 0)=0$ for all $t \in \mathbb{N}_{a}$ and there exists $\phi(r) \in \mathcal{K}$ such that $\phi(r) \leq V(t, x),\|x\|=r,(t, x) \in \mathbb{N}_{a} \times S_{\rho}$.

Definition 3.4. A real valued function $V(t, x)$ defined on $\mathbb{N}_{a} \times S_{\rho}$, where $S_{\rho}=\left\{x \in \mathbb{R}^{n}:\|x\| \leq\right.$ $\rho\}$, is said to be decrescent if and only if $V(t, 0)=0$ for all $t \in \mathbb{N}_{a}$ and there exists $\varphi(r) \in \mathcal{K}$ such that $V(t, x) \leq \varphi(r),\|x\|=r,(t, x) \in \mathbb{N}_{a} \times S_{\rho}$.

Now, we can state the theorems regarding the stability of solutions of the system (3.1).

Theorem 3.5. If there exists a positive definite and decrescent scalar function $V(t, x) \in C\left[\mathbb{N}_{a} \times\right.$ $\left.S_{\rho}, \mathbb{R}_{+}\right]$such that ${ }^{C} \Delta_{t_{0}}^{\alpha} V(t, x(t)) \leq 0$ for all $t_{0} \in \mathbb{N}_{a}$ and $(t, x) \in \mathbb{N}_{0} \times S_{\rho}$, then the trivial solution of (3.1) is uniformly stable.

Proof. Let $x(t)=x\left(t, t_{0}, x_{0}\right)$ be a solution of system (3.1). Since $V(t, x)$ is positive definite and decrescent, there exist $\phi, \varphi \in \mathcal{K}$ such that $\phi(\|x\|) \leq V(t, x) \leq \varphi(\|x\|)$ for all $(t, x) \in \mathbb{N}_{a} \times S_{\rho}$. For each $\epsilon>0,0<\epsilon<\rho$, we choose a $\delta=\delta(\epsilon)$ such that $\varphi(\delta)<\phi(\epsilon)$. For any solution $x(t)$ of (3.1) we have $\phi(\|x(t)\|) \leq V(t, x(t))$ with $\left\|x_{0}\right\|<\delta(\epsilon)$. Since $\Delta_{t_{0}}^{\alpha} V(t, x(t)) \leq 0$, by using (2.14) in Property 4 we have $V(t, x(t)) \leq V\left(t_{0}, x_{0}\right)$ for all $t \in \mathbb{N}_{t_{0}}$. Consequently,

$$
\phi(\|x(t)\|) \leq V(t, x(t)) \leq V\left(t_{0}, x_{0}\right) \leq \varphi\left(\left\|x_{0}\right\|\right)<\varphi(\delta)<\phi(\epsilon),
$$

and thus $\|x(t)\|<\epsilon$ for all $t \in \mathbb{N}_{t_{0}}$.

Theorem 3.6. If there exists a positive definite and decrescent scalar function $V(t, x) \in C\left[\mathbb{N}_{a} \times\right.$ $\left.S_{\rho}, \mathbb{R}_{+}\right]$such that

$$
{ }^{C} \Delta_{t_{0}}^{\alpha} V(t, x(t)) \leq-\psi(\|x(t+\alpha-1)\|), \quad \forall t_{0} \in \mathbb{N}_{a},(t, x) \in \mathbb{N}_{0} \times S_{\rho},
$$

where $\psi \in \mathcal{K}$, then the trivial solution of (3.1) is uniformly asymptotically stable. 
Proof. Since all the conditions of Theorem 3.5 are satisfied, the trivial solution of the system (3.1) is uniformly stable. Let $0<\epsilon<\rho$ and $\delta=\delta(\epsilon)$ correspond to uniform stability. Choose a fixed $\epsilon_{0} \leq \rho$ and $\delta_{0}=\delta\left(\epsilon_{0}\right)>0$. Now, choose $\left\|x_{0}\right\|<\delta_{0}$ and $T(\epsilon)$ large enough such that $(T+a)^{(\alpha)} \geq\left(\phi\left(\delta_{0}\right) / \psi(\delta(\epsilon))\right) \Gamma(\alpha+1)$. Such a large $T$ can be chosen since $\lim _{T \rightarrow \infty}(\Gamma(T+$ $\alpha) / \Gamma(T))=\infty$. Now, we claim that $\left\|x\left(t, t_{0}, x_{0}\right)\right\|<\delta(\epsilon)$ for all $t \in\left[t_{0}, t_{0}+T\right] \cap \mathbb{N}_{t_{0}}$. If this is not true, due to (3.3) and Property 4, we get

$$
\begin{aligned}
V\left(t, x\left(t, t_{0}, x_{0}\right)\right) & \leq V\left(t_{0}, x_{0}\right)-\frac{1}{\Gamma(\alpha)} \sum_{s=t_{0}+1-\alpha}^{t-\alpha}(t-\sigma(s))^{(\alpha-1)} \psi(\|x(s+\alpha-1)\|) \\
& \leq \phi\left(\left\|x_{0}\right\|\right)-\frac{\psi(\delta)}{\Gamma(\alpha)} \sum_{s=n_{0}}^{t-\alpha}(t-\sigma(s))^{(\alpha-1)} \\
& \leq \phi\left(\delta_{0}\right)-\frac{\psi(\delta)}{\Gamma(\alpha+1)}\left(t-n_{0}\right)^{(\alpha)}
\end{aligned}
$$

Substituting $t=t_{0}+T$, we get

$$
0<\varphi(\delta(\epsilon)) \leq V\left(t_{0}+T, x\left(t_{0}+T, t_{0}, x_{0}\right)\right) \leq \phi\left(\delta_{0}\right)-\frac{\psi(\delta)}{\Gamma(\alpha+1)}\left(T+t_{0}-n_{0}\right)^{(\alpha)} \leq 0
$$

which is a contradiction. Thus, there exists a $t \in\left[t_{0}, t_{0}+T\right]$ such that $\|x(t)\|<\delta(\epsilon)$. But in this case, since the trivial solution is uniformly stable and $t$ is arbitrary, $\|x(t)\|<\epsilon$ for all $t \geq t_{0}+T$ whenever $\left\|x_{0}\right\|<\delta_{0}$.

Theorem 3.7. If there exists a function $V(t, x) \in C\left[\mathbb{N}_{a} \times \mathbb{R}^{n}, \mathbb{R}_{+}\right]$such that

$$
\begin{gathered}
\phi(\|x(t)\|) \leq V(t, x) \leq \varphi(\|x(t)\|) \quad \forall(t, x) \in \mathbb{N}_{a} \times \mathbb{R}^{n}, \\
{ }^{C} \Delta_{t_{0}}^{\alpha} V(t, x(t)) \leq-\psi(\|x(t+\alpha-1)\|) \quad \forall t_{0} \in \mathbb{N}_{a},(t, x) \in \mathbb{N}_{0} \times \mathbb{R}^{n},
\end{gathered}
$$

where $\phi, \varphi$, and $\psi \in \mathcal{K} R$ hold for all $(t, x) \in \mathbb{N}_{a} \times \mathbb{R}^{n}$, then the trivial solution of (3.1) is globally uniformly asymptotically stable.

Proof. Since the conditions of Theorem 3.6 are satisfied, the trivial solution of (3.1) is uniformly asymptotically stable. It remains to show that the domain of attraction of $x=0$ is all of $\mathbb{R}^{n}$. Since $\lim _{r \rightarrow \infty} \phi(r)=\infty, \delta_{0}$ in the proof of Theorem 3.6 may be chosen arbitrary large and $\epsilon$ can be chosen such that it satisfies $\varphi\left(\delta_{0}\right)<\phi(\epsilon)$. Thus, the globally uniformly asymptotic stability of $x=0$ is concluded.

Lemma 3.8. If $V\left(t_{0}, x\left(t_{0}\right)\right) \geq 0$, then, for $0<\alpha \leq 1$, one has

$$
{ }^{C} \Delta_{t_{0}}^{\alpha} V(t, x(t)) \leq \Delta_{t_{0}}^{\alpha} V(t, x(t)) .
$$


Proof. From Property 3, we have

$$
{ }^{C} \Delta_{t_{0}}^{\alpha} V(t, x(t))=\Delta_{t_{0}}^{\alpha} V(t, x(t))-\frac{\left(t-t_{0}\right)^{(-\alpha)}}{\Gamma(1-\alpha)} V\left(t_{0}, x\left(t_{0}\right)\right) \quad \forall t \in \mathbb{N}_{t_{0}} .
$$

Since $V\left(t_{0}, x\left(t_{0}\right)\right) \geq 0$ and $\left(t-t_{0}\right)^{(-\alpha)} / \Gamma(1-\alpha) \geq 0$, we obtain

$$
{ }^{\mathrm{C}} \Delta_{t_{0}}^{\alpha} V(t, x(t)) \leq \Delta_{t_{0}}^{\alpha} V(t, x(t)) .
$$

Theorem 3.9. (i) If the assumptions in Theorem 3.5 are satisfied except replacing ${ }^{C} \Delta_{t_{0}}^{\alpha}$ by $\Delta_{t_{0}}^{\alpha}$, then the trivial solution of (3.1) is uniformly stable.

(ii) If the assumptions in Theorem 3.6 are satisfied except replacing ${ }^{C} \Delta_{t_{0}}^{\alpha}$ by $\Delta_{t_{0}}^{\alpha}$, then the trivial solution of (3.1) is uniformly asymptotically stable.

(iii) If the assumptions in Theorem 3.7 are satisfied except replacing ${ }^{C} \Delta_{t_{0}}^{\alpha}$ by $\Delta_{t_{0}}^{\alpha}$, then the trivial solution of (3.1) is globally uniformly asymptotically stable.

Proof. The proof is done by using Lemma 3.8 and following the same arguments as in the proofs of Theorem 3.5, Theorem 3.6, and Theorem 3.7.

\section{Conclusion}

In this paper using the Lyapunov direct method, we studied the stability of discrete nonautonomous systems in the sense of the Caputo fractional difference. We listed the conditions for uniform stability, uniformly asymptotic stability, and globally uniformly asymptotic stability for such systems. We will consider the Mittag-Leffler stability for such systems in future works.

\section{Acknowledgments}

This work is partially supported by the Scientific and Technical Research Council of Turkey. The authors would like to thank to the anonymous referee for her/his valuable comments.

\section{References}

[1] A. A. Kilbas, H. M. Srivastava, and J. J. Trujillo, Theory and Applications of Fractional Differential Equations, Elsevier Science B.V., Amsterdam, The Netherlands, 2006.

[2] I. Podlubny, Fractional Differential Equations, Academic Press, San Diego, Calif, USA, 1999.

[3] G. Samko, A. A. Kilbas, and O. I. Marichev, Fractional Integrals and Derivatives: Theory and Applications, Gordon and Breach Science, Yverdon, Switzerland, 1993.

[4] B. J. West, M. Bologna, and P. Grigolini, Physics of Fractal Operators, Springer-Verlag, New York, NY, USA, 2003.

[5] R. L. Magin, Fractional Calculus in Bioengineering, Begell House, Connecticut, Conn, USA, 2006.

[6] R. L. Bagley and P. J. Torvik, "On the appearance of the fractional derivative in the behavior of real materials," Journal of Applied Mechanics, Transactions ASME, vol. 51, no. 2, pp. 294-298, 1984. 
[7] K. S. Miller and B. Ross, "Fractional difference calculus," in Proceedings of the International Symposium on Univalent Functions, Fractional Calculus and Their Applications, pp. 139-152, Nihon University, Koriyama, Japan, 1989.

[8] F. M. Atici and P. W. Eloe, "A transform method in discrete fractional calculus," International Journal of Difference Equations, vol. 2, no. 2, pp. 165-176, 2007.

[9] F. M. Atici and P. W. Eloe, "Initial value problems in discrete fractional calculus," Proceedings of the American Mathematical Society, vol. 137, no. 3, pp. 981-989, 2009.

[10] F. M. Atic1 and P. W. Eloe, "Discrete fractional calculus with the nabla operator," Electronic Journal of Qualitative Theory of Differential Equations, no. 3, pp. 1-12, 2009.

[11] T. Abdeljawad and D. Baleanu, "Fractional differences and integration by parts," Journal of Computational Analysis and Applications, vol. 13, no. 3, pp. 574-582, 2011.

[12] T. Abdeljawad, "On Riemann and Caputo fractional differences," Computers $\mathcal{E}$ Mathematics with Applications, vol. 62, no. 3, pp. 1602-1611, 2011.

[13] J. Chen, D. M. Xu, and B. Shafai, “On sufficient conditions for stability independent of delay," Institute of Electrical and Electronics Engineers. Transactions on Automatic Control, vol. 40, no. 9, pp. 1675-1680, 1995.

[14] M. P. Lazarević, "Finite time stability analysis of fractional control of robotic time-delay systems," Mechanics Research Communications, vol. 33, no. 2, pp. 269-279, 2006.

[15] W. Deng, C. Li, and J. Lü, “Stability analysis of linear fractional differential system with multiple time delays," Nonlinear Dynamics, vol. 48, no. 4, pp. 409-416, 2007.

[16] F. Merrikh-Bayat and M. Karimi-Ghartemani, "An efficient numerical algorithm for stability testing of fractional-delay systems," ISA Transactions, vol. 48, no. 1, pp. 32-37, 2009.

[17] X. Zhang, "Some results of linear fractional order time-delay system," Applied Mathematics and Computation, vol. 197, no. 1, pp. 407-411, 2008.

[18] S. Momani and S. Hadid, "Lyapunov stability solutions of fractional integrodifferential equations," International Journal of Mathematics and Mathematical Sciences, no. 45 48, pp. 2503-2507, 2004.

[19] Y. Li, Y. Q. Chen, and I. Podlubny, "Stability of fractional-order nonlinear dynamic systems: Lyapunov direct method and generalized Mittag-Leffler stability," Computers \& Mathematics with Applications, vol. 59, no. 5, pp. 1810-1821, 2010.

[20] R. P. Agarwal, Difference Equations and Inequalities, Theory, Methods, and Application, Marcel Dekker, New York, NY, USA, 2000. 


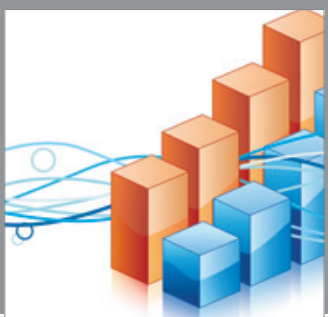

Advances in

Operations Research

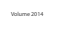

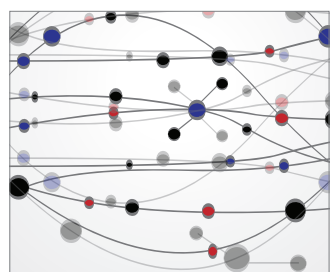

\section{The Scientific} World Journal
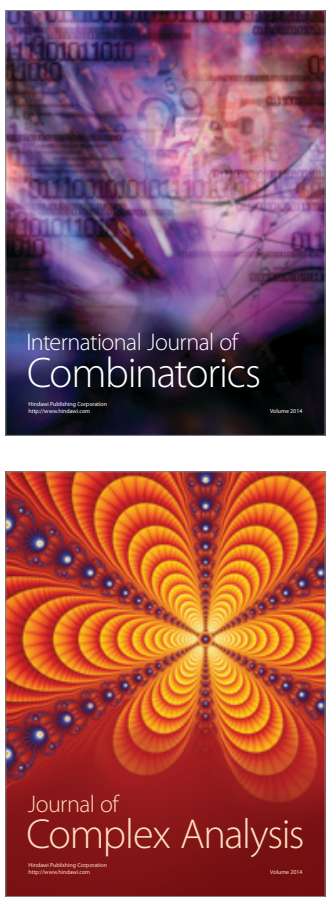

International Journal of

Mathematics and

Mathematical

Sciences
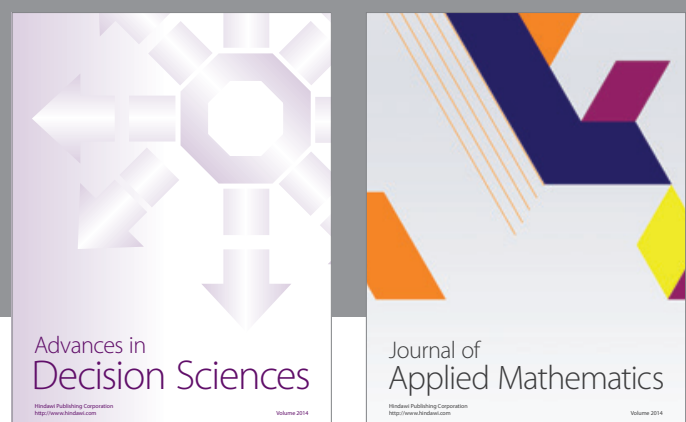

Journal of

Applied Mathematics
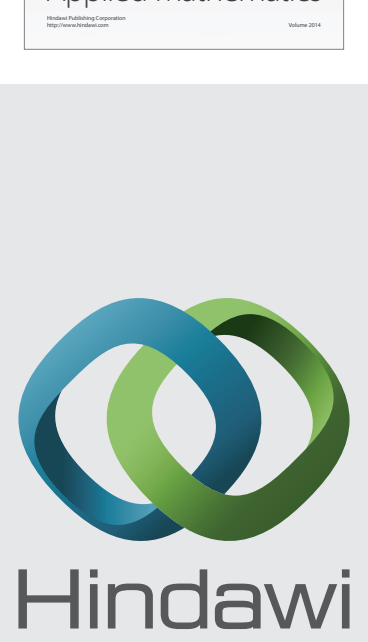

Submit your manuscripts at http://www.hindawi.com
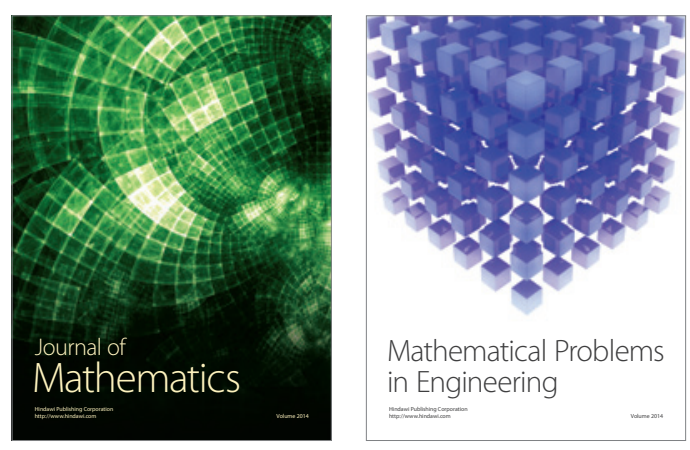

Mathematical Problems in Engineering
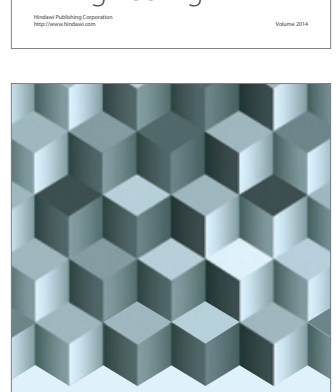

Journal of

Function Spaces
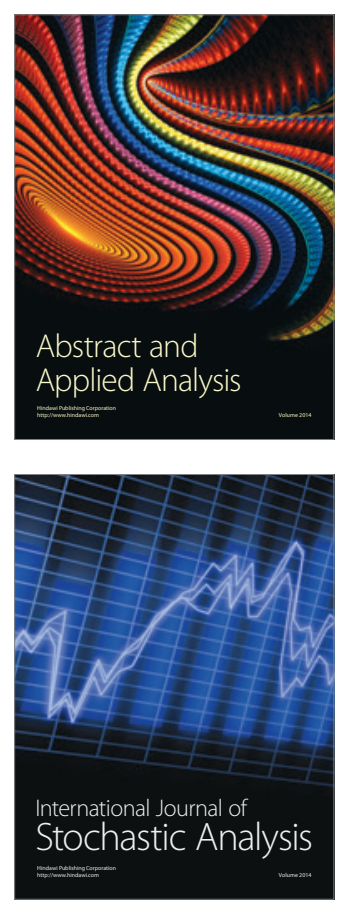

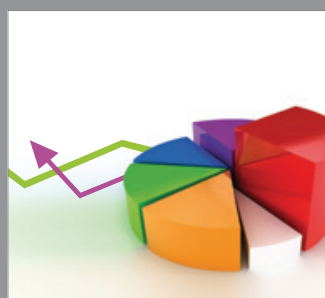

ournal of

Probability and Statistics

Promensencen
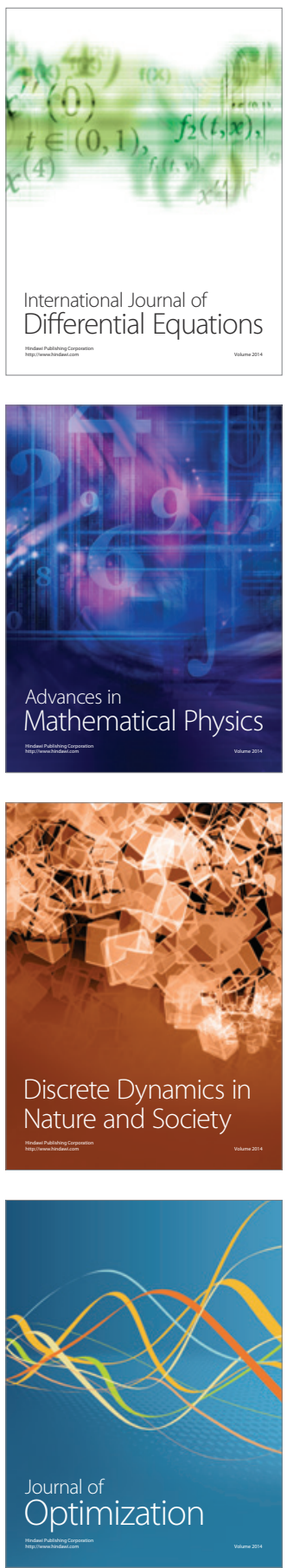